\title{
SPL Business Entity Qualifier Terminology
}

National Cancer Institute

\section{Source}

National Cancer Institute. SPL Business Entity Qualifier Terminology. NCI Thesaurus.

Code $C 117110$.

Terminology used in the framework of identifying types of business entities. 